Research Article

\title{
Value of Urinary Neutrophil Gelatinase-Associated Lipocalin versus Conventional Biomarkers in Predicting Response to Treatment of Active Lupus Nephritis
}

\author{
Mohamed Abd El-Mohsen (1D, Ahmed Tawfik, Walid Bichari, Sahar Shawky, \\ Gamal Mady, and Mohamed Hassan \\ Internal Medicine and Nephrology Department, Faculty of Medicine, Ain Shams University, Cairo, Egypt \\ Correspondence should be addressed to Mohamed Abd El-Mohsen; mohamedatef99@hotmail.com
}

Received 31 July 2020; Revised 28 August 2020; Accepted 7 September 2020; Published 7 October 2020

Academic Editor: David B. Kershaw

Copyright (c) 2020 Mohamed Abd El-Mohsen et al. This is an open access article distributed under the Creative Commons Attribution License, which permits unrestricted use, distribution, and reproduction in any medium, provided the original work is properly cited.

\begin{abstract}
Introduction. Lupus nephritis (LN) affects almost two-thirds of systemic lupus erythematosus (SLE) patients. Despite initial aggressive therapy, up to $25 \%$ of patients with $\mathrm{LN}$ will progress to permanent renal damage. Conventional serum markers for LN lack the sensitivity of an ideal biomarker. Urinary neutrophil gelatinase-associated lipocalin (UNGAL) is an excellent biomarker for early diagnosis of acute kidney injury and predicting renal outcomes. Objective. To measure UNGAL among LN patients to correlate its levels with renal disease activity and to investigate its predictive performance in response to induction therapy. Patients and Methods. 40 SLE patients with biopsy-proven LN class III, IV, or V were randomly selected. The study was conducted in the internal medicine department and outpatient clinic in Ain Shams University Hospitals and completed after six months. UNGAL was measured at baseline, three-month follow-up, and after complete induction therapy. Results. In LN patients at baseline, the mean serum creatinine was $2.57 \pm 0.96 \mathrm{mg} / \mathrm{dL}$ and the mean UNGAL was $33.50 \pm 18.34 \mathrm{ng} / \mathrm{dL}$. Mean UNGAL levels of complete response, partial response, and nonresponse groups were $14.48 \pm 2.99 \mathrm{ng} / \mathrm{mL}, 34.49 \pm 4.09 \mathrm{ng} / \mathrm{mL}$, and $62.07 \pm 14.44 \mathrm{ng} / \mathrm{mL}$, respectively. Based on the ROC curve, we found a better performance of baseline UNGAL to discriminate the complete response group from partial and nonresponse groups to predict response to induction, outperforming conventional biomarkers. The area under the curve was 0.943 , and the best cutoff level was $26.5 \mathrm{ng} / \mathrm{mL}(92.31 \%$ sensitivity and $88.89 \%$ specificity). Conclusion. UNGAL performed better than conventional biomarkers in predicting response to treatment of active LN.
\end{abstract}

\section{Introduction}

SLE is a challenging condition that has an unpredictable course that presents unique issues in diagnosis and management [1]. It mostly affects women during the childbearing period, up to $20 \%$ of cases being affected in childhood; it is characterized by loss of self-tolerance and development of autoantibodies (autoAbs) to nuclear selfantigens [2]. SLE diagnosis is based on characteristic clinical manifestations affecting joints, skin, central nervous system, and the kidneys along with serological markers [3]. The basis for diagnosis was established by the American College of Rheumatology (ACR) based on the presence of any four out of eleven criteria which were recently revised in 2015 [4]. LN is a major risk factor for morbidity and mortality in SLE, and $10 \%$ of patients with LN will develop end-stage renal disease (ESRD) [5]. Evaluation is straightforward and should include a urinalysis and measurement of kidney function, generally serum creatinine concentration or estimated glomerular filtration rate (eGFR) [6]. Renal biopsy is the gold standard in the diagnosis and classification of LN [7]. Renal biopsy, although considered to be a benign procedure, sometimes can have serious complications, even in expert hands, a reason why this cannot be performed repeatedly to assess renal status. Therefore, the goal of studying newer LN biomarkers is to overcome the drawbacks of the existing 
biomarkers and replace the need for renal biopsy [8]. Tubulointerstitial damage is important in predicting the progression of glomerular diseases and LN [9]. Neutrophil gelatinase-associated lipocalin (NGAL) is a $25-\mathrm{kD}$ protein secreted by leukocytes and tubular epithelial cells under different conditions of stress or inflammation [10]. NGAL is widely expressed following ischemic or nephrotoxic injury in humans. NGAL is thought to mediate inflammatory responses by sequestering neutrophil chemoattractants such as leukotriene B4 and platelet-activating factor [11]. Several clinical studies found that UNGAL represented a very sensitive and highly predictive biomarker for progressive tubular and glomerular injury [12]. Furthermore, in a previous study on SLE patients, an increase in UNGAL levels correlated with renal disease activity [13]. In the current study, we hypothesized that urinary NGAL would significantly correlate with the severity of renal disease activity and might predict the renal response to induction therapy.

\section{Objective}

The objective of this study was to measure UNGAL among LN patients to correlate its levels with the severity of renal disease activity and investigate its predictive performance in the renal response to induction therapy.

\subsection{Patients and Methods}

2.1.1. Study Population. Our study included 40 SLE patients with biopsy-proven LN class III, IV, or V. Patients fulfilled at least four of the ACR 1982 revised criteria to diagnose SLE. All patients were randomly selected from the internal medicine department and outpatient clinic in Ain Shams University Hospitals during 2018 and 2019. We excluded diabetic patients, patients on hemodialysis, renal transplant patients, and patients with nonlupus-related renal affection, drug-induced lupus, overlapping syndromes, urinary tract infection, active systemic infection, and active malignancies. All patients received standard induction therapy. Patients were divided into three groups based on the renal response to treatment. Patients with complete response were defined by the return of serum creatinine to the previous baseline plus a decline in urinary protein-to-creatinine ratio (UPCR) to $<500 \mathrm{mg} / \mathrm{g}$. Partial response was defined by stabilization or improved serum creatinine level plus a $\geq 50 \%$ decrease in UPCR. The nonresponse group of patients was defined by a sustained increase in serum creatinine level or a $<50 \%$ decrease in UPCR.

2.1.2. Treatment of Lupus Nephritis. Induction of remission for active LN was performed using a combination of pulse intravenous (IV) methylprednisolone and either pulse IV cyclophosphamide (CYC) or oral mycophenolate mofetil (MMF). Pulse IV methylprednisolone therapy regimen ranged from 500 to $1000 \mathrm{mg}$ IV daily for three consecutive days. Six pulses of intravenous cyclophosphamide $\left(0.5-1 \mathrm{~g} / \mathrm{m}^{2}\right)$ on consecutive months were offered to patients or MMF $(2-3 \mathrm{~g} / \mathrm{d}$ for six months) especially for women with childbearing potential.
Maintenance dose of oral prednisolone $0.5-1 \mathrm{mg} / \mathrm{kg}$ per day was tapered slowly over a few weeks to the lowest effective dose.

2.1.3. Clinical and Laboratory Measurement. All participants were subjected to detailed history taking, including sociodemographic history (age, sex, residence, and marital status), stressing on the presence or absence of constitutional symptoms (fever, malaise, myalgia, or weight loss), significant hair loss, skin rash, oral ulcers, and photosensitivity. An assessment was carried out for joint-, pulmonary-, cardiovascular-, renal-, hematological-, and neuropsychiatric-related complaints. Careful drug, menstrual, and family histories were taken. A thorough systemic physical examination was performed, including blood pressure measurement and skin and mucosal examination. Cardiac, rheumatological, and neurological examinations were performed. Assessment of disease activity by the SLE disease activity index (SLEDAI) scoring system [14] was carried out for each patient (the minimum score is 0 and the maximum is 105). Renal involvement was assessed by a renal SLEDAI (rSLEDAI) score of 4 (the minimum score is 0 and the maximum is 16), corresponding to the presence of any one of the following concerning urine analysis: hematuria, proteinuria, pyuria, or urinary red cell casts. Renal histological features were evaluated by a renal pathologist. Activity index scores were calculated from the summation of individual scores. The range of activity index score was 0 to 24 with higher scores representing higher activity. Chronicity index scores were calculated from the summation of individual scores. The range of chronicity index score was 0 to 12 with higher scores representing higher chronicity [15]. Blood samples were obtained at baseline, three months after induction, and after complete induction to determine complete blood cell count, serum creatinine level, blood urea nitrogen (BUN), $\mathrm{C} 3$ and $\mathrm{C} 4$ fractions of the complement, anti-nuclear antibodies (ANA), and anti-dsDNA antibody. Estimated glomerular filtration rate (eGFR) was assessed using the Cockcroft-Gault equation. Complete urinalysis and UPCR were performed.

\subsubsection{NGAL Enzyme-Linked Immunosorbent Assay.} Urine samples were collected at baseline, three months after induction, and after complete induction, centrifuged at 2000-3000 rpm for approximately 20 minutes to remove particular impurities, and then stored frozen at $-80^{\circ} \mathrm{C}$ until assayed. The UNGAL level was measured by the Biont human NGAL enzyme-linked immunosorbent assay (ELISA) kit (catalog no. YLA0724HU) which is a sandwich enzyme immune assay for in vitro qualitative measurement of NGAL. All measurements were made in triplicate and blinded manner. Urinary NGAL excretions were reported as the amount of urinary NGAL in nanograms per milliliter (ng/mL).

2.1.5. Ethical Issues. The research followed the tenets of the Declaration of Helsinki. The research was approved by the Ethics Committee of Faculty of Medicine of Ain 
Shams University (FWA 000017585). Informed consents were obtained from all patients who participated in this study.

2.1.6. Statistical Methods. Data were revised for its completeness and consistency. Double data entry was done on IBM SPSS Statistics version 23.0 (IBM Corp., Armonk, NY, USA). Quantitative data were summarized by mean along with standard deviation, while qualitative data were summarized by numbers and percentages. Student's $t$-test was used to compare quantitative data between two independent groups, and the one-way analysis of variance (ANOVA) test was used for more than two groups. A chi-square test was used to compare qualitative data between different groups. The repeated measures ANOVA test was used to compare quantitative data for the same group at different time points, and the Friedman test was used for qualitative data. Pearson's correlation test was used to measure the correlation between different continuous variables. Receiver operating characteristic (ROC) analysis was used to calculate the area under the curve (AUC) with associated 95\% confidence interval (CI) for UNGAL and conventional biomarkers that were used to predict renal response and to find the best cutoff values to identify the renal response after induction therapy. A " $p$ value" of less than 0.05 was considered statistically significant.

\section{Results}

A total of 40 patients were included in this study, most patients were females (87.5\%), with a mean age of $25.63 \pm 4.26$ years. The duration of SLE ranged from recently diagnosed patients up to 10 years. About $20 \%$ of patients had constitutional symptoms of SLE. About $20 \%$ of patients had nephritis only, and the rest of the patients had systemic organ involvement in addition to nephritis. Disease activity that was assessed using the SLEDAI score was $19.55 \pm 4.70$. Renal activity assessed using the rSLEDAI score was $14.20 \pm 3.13$. Most of the patients were classified as LN class IV (45\%). Coclassification with class V was found among nine patients (22.5\%). Mean scores of activity index and chronicity index in biopsy were $7.53 \pm 1.55$ and $4.75 \pm 1.21$, respectively. At baseline, the mean UPCR was $2.89 \pm 1.39 \mathrm{~g} / \mathrm{g}$ creatinine, mean serum creatinine was $2.57 \pm 0.96 \mathrm{mg} / \mathrm{dL}$, and mean UNGAL was $33.50 \pm 18.34 \mathrm{ng} / \mathrm{mL}$. The patients' characteristics and laboratory results are described in Table 1.

\subsection{Baseline UNGAL with Patients' Characteristics and} Laboratory Results. Among LN patients, we found no relation between UNGAL with age, sex, duration of disease, systemic organ involvement, and different classes of LN. The UNGAL level correlated positively with rising blood pressure (systolic and diastolic) $(p=0.01)$; moreover, positive correlations were observed between baseline UNGAL and each of the following: SLEDAI score $(p=0.01)$, chronicity index $(p=0.001), \mathrm{BUN}$, and serum creatinine $(p<0.001)$. UNGAL correlated negatively with
TABLE 1: Patients' characteristics and laboratory results.

\begin{tabular}{|c|c|}
\hline Variable & No. (\%) or mean/SD \\
\hline Female & $35(87.5 \%)$ \\
\hline Age (years) & $25.63 \pm 4.26$ \\
\hline Duration of SLE (months) & $37 \pm 29.5$ \\
\hline Systolic blood pressure $(\mathrm{mmHg})$ & $130.00 \pm 11.98$ \\
\hline Diastolic blood pressure (mmHg) & $75.00 \pm 9.94$ \\
\hline SLEDAI score & $19.55 \pm 4.70$ \\
\hline rSLEDAI score & $14.20 \pm 3.13$ \\
\hline \multicolumn{2}{|l|}{ Systemic organ involvement } \\
\hline Nephritis only & $8(20.0 \%)$ \\
\hline Arthritis & $11(27.5 \%)$ \\
\hline Cutaneous lupus & $11(27.5 \%)$ \\
\hline Serositis & $11(27.5 \%)$ \\
\hline Hematological involvement & $9(22.5 \%)$ \\
\hline Carditis & $4(10 \%)$ \\
\hline Vasculitis & $2(5 \%)$ \\
\hline Neurological lupus & $2(5 \%)$ \\
\hline \multicolumn{2}{|l|}{ ISN/RPS class } \\
\hline III & $5(12.5 \%)$ \\
\hline IV & $18(45.0 \%)$ \\
\hline $\mathrm{V}$ & $4(10.0 \%)$ \\
\hline $\mathrm{III}+\mathrm{V}$ & $4(10.0 \%)$ \\
\hline $\mathrm{IV}+\mathrm{V}$ & $9(22.5 \%)$ \\
\hline Activity index in renal biopsy & $7.53 \pm 1.55$ \\
\hline Chronicity index in renal biopsy & $4.75 \pm 1.21$ \\
\hline \multicolumn{2}{|l|}{ Immunosuppressive agents } \\
\hline IV cyclophosphamide & $25(62.5 \%)$ \\
\hline Mycophenolate mofetil & $15(37.5 \%)$ \\
\hline \multicolumn{2}{|l|}{ Laboratory results } \\
\hline Serum albumin & $2.56 \pm 0.37 \mathrm{~g} / \mathrm{dL}$ \\
\hline Hemoglobin & $9.89 \pm 1.46 \mathrm{gm} / \mathrm{dL}$ \\
\hline BUN & $46.53 \pm 8.86 \mathrm{mg} / \mathrm{dL}$ \\
\hline Creatinine & $2.57 \pm 0.96 \mathrm{mg} / \mathrm{dL}$ \\
\hline eGFR & $\begin{array}{c}41.63 \pm 26.02 \mathrm{~mL} / \mathrm{min} / \\
1.73 \mathrm{~m}^{2}\end{array}$ \\
\hline Complement component 3 (C3) & $63.90 \pm 19.65 \mathrm{mg} / \mathrm{dL}$ \\
\hline Complement component 4 (C4) & $16.75 \pm 4.53 \mathrm{mg} / \mathrm{dL}$ \\
\hline $\mathrm{Pr} / \mathrm{Cr}$ ratio & $2.89 \pm 1.39 \mathrm{mg} / \mathrm{mg}$ \\
\hline UNGAL & $33.50 \pm 18.34 \mathrm{ng} / \mathrm{mL}$ \\
\hline
\end{tabular}

SLE, systemic lupus erythematosus; SLEDAI, SLE disease activity index; rSLEDAI, renal SLE disease activity index; ISN/RPS, International Society of Nephrology/Renal Pathology Society; IV, intravenous; eGFR, estimated glomerular filtration rate; BUN, blood urea nitrogen; C3, complement component 3; $\mathrm{C} 4$, complement component 4; $\mathrm{Pr} / \mathrm{Cr}$ ratio, protein-tocreatinine ratio; UNGAL, urinary neutrophil gelatinase-associated lipocalin.

complement C3 $(p=0.01)$, while no correlations were found regarding UNGAL with proteinuria and rSLEDAI score; Table 2.

3.2. Patients' Characteristics and Baseline Laboratories in Different Groups (Classified according to Renal Response to Induction). Patients received induction of remission, both clinical and laboratory parameters improved. We found a significant reduction in UNGAL levels after three months and after complete induction. Upon classifying the patients into three groups according to renal response to treatment, we found 13 patients with complete response, 19 patients with partial response, and eight patients with no response to 
TABLE 2: Correlation of baseline UNGAL with demographic, clinical, and laboratory data of patients.

\begin{tabular}{lcc}
\hline \multirow{2}{*}{ Variable } & \multicolumn{2}{c}{ UNGAL } \\
\hline Age & $r^{*}$ & $p$ value \\
Duration of SLE & 0.116 & 0.477 \\
Systolic blood pressure & 0.02 & 0.91 \\
Diastolic blood pressure & 0.42 & 0.01 \\
SLEDAI score & 0.43 & 0.01 \\
rSLEDAI score & 0.40 & 0.01 \\
Activity index in biopsy & 0.30 & 0.06 \\
Chronicity index in biopsy & 0.12 & 0.45 \\
Serum albumin & 0.49 & 0.001 \\
Hemoglobin & 0.23 & 0.15 \\
Baseline complement 3 & -0.25 & 0.12 \\
Baseline complement 4 & -0.42 & 0.01 \\
BUN & -0.17 & 0.30 \\
Creatinine & 0.57 & $<0.001$ \\
eGFR & 0.72 & $<0.001$ \\
Pr/Cr ratio & -0.57 & $<0.001$ \\
\hline
\end{tabular}

${ }^{*}$ Pearson's correlation: significant at 0.05 . SLE, systemic lupus erythematosus; SLEDAI, SLE disease activity index; rSLEDAI, renal SLE disease activity index; eGFR, estimated glomerular filtration rate; BUN, blood urea nitrogen; $\mathrm{Pr} / \mathrm{Cr}$ ratio, protein-to-creatinine ratio; UNGAL, urinary neutrophil gelatinase-associated lipocalin.

induction therapy. Baseline UNGAL levels were significantly lower among patients with complete response $(14.48 \pm 2.99 \mathrm{ng} / \mathrm{mL})$ than those with partial response $(34.49 \pm 4.09 \mathrm{ng} / \mathrm{mL})$ and nonresponse $(62.07 \pm 14.44 \mathrm{ng} / \mathrm{mL})$ $(p<0.001)$. We did not find a significant difference between the three groups as regards gender, age, disease duration, LN class, SLEDAI, and rSLEDAI scores. Significantly higher blood pressure (systolic and diastolic) was observed in the nonresponse group compared to the complete response group $(p=0.03$ and $p=0.02)$. As expected, patients with no response had significantly higher renal chronicity index $(p<0.001)$, while there were no significant differences regarding the renal activity index. We found significantly higher proteinuria in patients with complete renal response compared to the partial response group of patients $(p=0.002)$; moreover, patients with complete renal response had significantly lower serum creatinine and higher estimated GFR $(p<0.001)$. However, we found no significant differences in baseline complement levels among the three groups (Table 3).

\subsection{Predictive Performance of Urine NGAL versus Conven-} tional Biomarkers. ROC analysis was performed to calculate the AUC with associated 95\% CI comparing UNGAL and conventional biomarkers at baseline and three months after induction therapy, thus investigating the diagnostic reliability of UNGAL as a predictor of the renal response to induction therapy and differentiating the complete response group from partial and nonresponse groups. We found a better performance of baseline UNGAL to discriminate the complete response group from partial and nonresponse groups, outperforming conventional biomarkers. The area under the curve value was 0.943 , and the best cutoff value was $26.5 \mathrm{ng} / \mathrm{mL}$ $($ sensitivity $=92.31 \%$ and specificity $=88.89 \%)($ Tables 4 and 5 ;
Figure 1). After three months, when compared to other conventional biomarkers, UNGAL was found with an area under the curve value of 0.966 versus creatinine with an area under the curve value of 0.966 and eGFR with an area under the curve value of 0.979 . The best cutoff value for the three-month UNGAL was $17.4 \mathrm{ng} / \mathrm{mL}$ (sensitivity $=92.30 \%$ and specificity $=96.30 \%)$ (Tables 6 and 7; Figure 2).

\section{Discussion}

Biomarkers help in accurate evaluation of disease activity and enable the physician to individualize therapy. Biomarkers for LN should be different from the biomarkers of overall disease activity as LN requires significant immunosuppression. Consequently, prediction of renal activity and correlation with renal histological findings are needed in such biomarkers [8]. Despite the previous studies that were conducted to reveal the underlying mechanisms responsible for the pathogenesis of SLE, few biomarkers have been remarkably discovered. The lack of reliable biomarkers not only delays the clinical management of SLE but also delays the development of a newer therapeutic agent [16]. NGAL has emerged as a novel biomarker of great value for the diagnosis of kidney injury. NGAL is present in neutrophils, where it is bound with gelatin and usually expressed by the injured epithelia [17]. Previous studies have been conducted to evaluate the role of NGAL in the evaluation of kidney injury in patients with lupus nephritis $[18,19]$. In the present study, we investigated the performance of UNGAL in predicting treatment outcomes compared to conventional biomarkers of LN disease activity. Our study results indicated that UNGAL might be a novel biomarker of LN. Findings from the present study indicate that urinary NGAL might be a biomarker of the renal response to induction therapy outperforming serum creatinine and other conventional biomarkers. Our study showed that baseline UNGAL did not correlate with age, gender, and systemic organ involvement which is in agreement with the study by Satirapoj et al. [20] and El Shahawy et al. [21]. As regards the relation between baseline UNGAL and ISN/RPS class, we found no significant differences in baseline UNGAL between different classes of LN which is in agreement with the study by Alharazy et al. [22]. Our study showed that baseline UNGAL correlated with disease activity as it correlated positively with rising blood pressure (systolic and diastolic) and negatively with $\mathrm{C} 3$ which is in agreement with the study by Satirapoj et al. [20]. We found positive correlations between baseline UNGAL and each of the following: chronicity index, SLEDAI score (which possibly might reflect the renal components of the SLEDAI score and worsening renal function), BUN, and serum creatinine. However, we found no correlation between baseline UNGAL and rSLEDAI score; a possible explanation might be that all patients in our study had nephritis proven by biopsy with almost comparable renal affection and rSLEDAI scores among complete, partial, and nonresponse groups. Our results agreed with those of Batool et al. [23] as regards the positive correlation 
TABLE 3: Baseline characteristics and laboratory data of different groups (classified according to renal response to induction).

\begin{tabular}{|c|c|c|c|c|c|}
\hline Variable & $\begin{array}{l}\text { Complete response }(N=13) \\
\text { Mean/SD }\end{array}$ & $\begin{array}{c}\text { Partial response }(N=19) \\
\text { Mean/SD }\end{array}$ & $\begin{array}{c}\text { Nonresponse }(N=8) \\
\text { Mean/SD }\end{array}$ & $\mathrm{F}^{*}$ & $p$ value \\
\hline Baseline SLEDAI & $18.92 \pm 4.21$ & $18.74 \pm 5.04$ & $22.50 \pm 3.82$ & 2.090 & 0.14 \\
\hline Baseline rSLEDAI & $13.54 \pm 3.84$ & $13.89 \pm 3.09$ & $16.00 \pm .00$ & 1.769 & 0.19 \\
\hline Activity index in biopsy & $7.38 \pm 1.45$ & $7.47 \pm 1.74$ & $7.88 \pm 1.36$ & 0.257 & 0.78 \\
\hline Chronicity index in biopsy & $4.08 \pm 0.76$ & $4.63 \pm 1.01$ & $6.13 \pm 1.25$ & 10.870 & $<0.001^{\mathrm{bc}}$ \\
\hline Hemoglobin & $10.62 \pm 1.68 \mathrm{~g} / \mathrm{dL}$ & $9.37 \pm 1.39 \mathrm{~g} / \mathrm{dL}$ & $9.95 \pm 0.58 \mathrm{~g} / \mathrm{dL}$ & 3.131 & 0.06 \\
\hline Serum albumin & $2.31 \pm 0.37 \mathrm{~g} / \mathrm{dL}$ & $2.73 \pm 0.32 \mathrm{~g} / \mathrm{dL}$ & $2.59 \pm 0.24 \mathrm{~g} / \mathrm{dL}$ & 6.488 & $0.004^{\mathrm{a}}$ \\
\hline $\mathrm{C} 3$ & $68.23 \pm 24.93 \mathrm{mg} / \mathrm{dL}$ & $64.58 \pm 18.04 \mathrm{mg} / \mathrm{dL}$ & $55.25 \pm 11.26 \mathrm{mg} / \mathrm{dL}$ & 1.108 & 0.34 \\
\hline $\mathrm{C} 4$ & $16.54 \pm 5.46 \mathrm{mg} / \mathrm{dL}$ & $17.05 \pm 4.08 \mathrm{mg} / \mathrm{dL}$ & $16.38 \pm 4.47 \mathrm{mg} / \mathrm{dL}$ & 0.080 & 0.92 \\
\hline BUN & $38.54 \pm 8.85 \mathrm{mg} / \mathrm{dL}$ & $49.63 \pm 6.17 \mathrm{mg} / \mathrm{dL}$ & $52.13 \pm 4.97 \mathrm{mg} / \mathrm{dL}$ & 13.011 & $<0.001^{\mathrm{ab}}$ \\
\hline Creatinine & $1.76 \pm 0.97 \mathrm{mg} / \mathrm{dL}$ & $2.69 \pm 0.57 \mathrm{mg} / \mathrm{dL}$ & $3.57 \pm 0.52 \mathrm{mg} / \mathrm{dL}$ & 16.349 & $<0.001^{\mathrm{abc}}$ \\
\hline eGFR & $\begin{array}{c}63.69 \pm 36.07 \mathrm{~mL} / \mathrm{min} / \\
1.73 \mathrm{~m}^{2}\end{array}$ & $\begin{array}{c}32.95 \pm 7.57 \mathrm{~mL} / \mathrm{min} / \\
1.73 \mathrm{~m}^{2}\end{array}$ & $\begin{array}{c}26.38 \pm 4.37 \mathrm{~mL} / \mathrm{min} / \\
1.73 \mathrm{~m}^{2}\end{array}$ & 10.607 & $<0.001^{\mathrm{abc}}$ \\
\hline $\mathrm{Pr} / \mathrm{Cr}$ ratio & $3.95 \pm 1.65 \mathrm{mg} / \mathrm{mg}$ & $2.30 \pm 0.96 \mathrm{mg} / \mathrm{mg}$ & $2.57 \pm 0.78 \mathrm{mg} / \mathrm{mg}$ & 7.549 & $0.002^{\mathrm{a}}$ \\
\hline UNGAL & $14.48 \pm 2.99 \mathrm{ng} / \mathrm{mL}$ & $34.49 \pm 4.09 \mathrm{ng} / \mathrm{mL}$ & $62.07 \pm 14.44 \mathrm{ng} / \mathrm{mL}$ & 111.428 & $<0.001^{\mathrm{abc}}$ \\
\hline
\end{tabular}

${ }^{*}$ One-way ANOVA test (post hoc test: ${ }^{a}$ complete response vs. partial response/ ${ }^{b}$ complete response vs. nonresponse/ ${ }^{c}$ partial response vs. nonresponse): significant at 0.05 level. SLE, systemic lupus erythematosus; SLEDAI, SLE disease activity index; rSLEDAI, renal SLE disease activity index; eGFR, estimated glomerular filtration rate; $\mathrm{BUN}$, blood urea nitrogen; $\mathrm{C}$, complement component 3; $\mathrm{C} 4$, complement component 4; $\mathrm{Pr} / \mathrm{Cr}$ ratio, protein-to-creatinine ratio; UNGAL, urinary neutrophil gelatinase-associated lipocalin.

TABLE 4: Comparison between complete responders with partial and nonresponders as regards to baseline renal biomarkers.

\begin{tabular}{lccc}
\hline Variable & Complete responders $(N=13)$ & Partial and nonresponders $(N=27)$ & Test value* \\
& Mean \pm SD & Mean \pm SD & -0.966 \\
C3 & $68.23 \pm 24.93 \mathrm{mg} / \mathrm{dL}$ & $61.81 \pm 16.68 \mathrm{mg} / \mathrm{dL}$ & 0.203 \\
C4 & $16.54 \pm 5.46 \mathrm{mg} / \mathrm{dL}$ & $16.85 \pm 4.12 \mathrm{mg} / \mathrm{dL}$ & 4.486 \\
Creatinine & $1.76 \pm 0.97 \mathrm{mg} / \mathrm{dL}$ & $2.95 \pm 0.68 \mathrm{mg} / \mathrm{dL}$ & 0.841 \\
eGFR & $63.69 \pm 36.07 \mathrm{~mL} / \mathrm{min} / 1.73 \mathrm{~m}^{2}$ & $31.00 \pm 7.36 \mathrm{~mL} / \mathrm{min} / 1.73 \mathrm{~m}^{2}$ & 0.0001 \\
Pr/Cr ratio & $3.95 \pm 1.65 \mathrm{mg} / \mathrm{mg}$ & $2.38 \pm 0.91 \mathrm{mg} / \mathrm{mg}$ & -4.575 \\
UNGAL & $17.35 \pm 7.22 \mathrm{ng} / \mathrm{mL}$ & $39.55 \pm 16.28 \mathrm{ng} / \mathrm{mL}$ & 0.0001 \\
\hline
\end{tabular}

*Student's $t$-test: significant at 0.05 level. C3, complement component 3; C4, complement component 4; eGFR, estimated glomerular filtration rate; Pr/Cr ratio, protein-to-creatinine ratio; UNGAL, urinary neutrophil gelatinase-associated lipocalin.

TABLE 5: Cutoff levels for baseline UNGAL and conventional biomarkers in predicting renal response to induction.

\begin{tabular}{|c|c|c|c|c|c|c|}
\hline Variable & AUC & Cutoff point & Sensitivity & Specificity & PPV & NPV \\
\hline Creatinine & 0.853 & $1.9 \mathrm{mg} / \mathrm{dL}$ & 76.92 & 92.59 & 83.3 & 89.3 \\
\hline eGFR & 0.806 & $36 \mathrm{~mL} / \mathrm{min} / 1.73 \mathrm{~m}^{2}$ & 84.62 & 77.78 & 64.7 & 91.3 \\
\hline $\mathrm{Pr} / \mathrm{Cr}$ ratio & 0.802 & $3 \mathrm{mg} / \mathrm{mg}$ & 76.92 & 77.78 & 62.5 & 87.5 \\
\hline UNGAL & 0.943 & $26.5 \mathrm{ng} / \mathrm{mL}$ & 92.31 & 88.89 & 80.0 & 96.0 \\
\hline
\end{tabular}

eGFR, estimated glomerular filtration rate; $\mathrm{Pr} / \mathrm{Cr}$ ratio, protein-to-creatinine ratio; UNGAL, urinary neutrophil gelatinase-associated lipocalin.

between baseline UNGAL and serum creatinine, while we found no correlation between baseline UNGAL and proteinuria which is in contrast to the study by Satirapoj et al. [20]. In addition, the current study did not find significant correlations between baseline UNGAL and other laboratory parameters, including $\mathrm{Hb}$, WBCs, platelets, and C4, which is in agreement with the study by El Shahawy et al. [21]. Upon classifying patients into three groups according to renal response to treatment to clarify the relationship between UNGAL and renal response, we found that baseline UNGAL levels were significantly lower among those with complete response than those with partial response with the highest baseline urine NGAL levels and those in the nonresponse group; these results agreed with those of El Shahawy et al. [21] and Satirapoj et al. [20], providing a strong evidence for
UNGAL as a predictor for treatment response. As regards renal chronicity index in the biopsy, it was significantly higher in patients with no response compared to patients with complete response and partial response, while no significant differences regarding renal activity index among the three groups, this goes in hand with the study by Satirapoj et al. [20]. Surprisingly, regarding proteinuria, we found that patients with complete renal response had significantly higher proteinuria. This finding could be explained by the heavy proteinuria occurred in class $\mathrm{V}$ in the complete response group of patients. We found significantly lower serum creatinine and higher estimated GFR in patients with complete renal response, in contrast to the study by Satirapoj et al. [20]. Regarding baseline complement, we found no significant differences between the three groups, unlike the study by Satirapoj et al. [20]. 


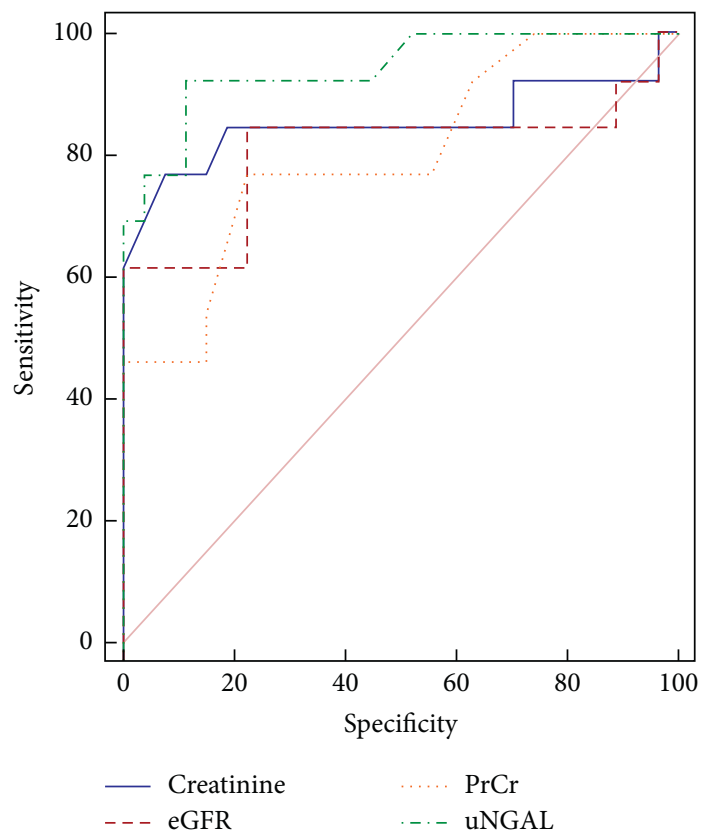

FIGURE 1: Graph ROC curves showing area under the curve of baseline UNGAL and conventional biomarkers to predict renal response to induction. It showed an AUC value of 0.943, outperforming conventional biomarkers.

TABLE 6: Comparison between complete responders with partial and nonresponders as regards three-month follow-up renal biomarkers.

\begin{tabular}{lccr}
\hline Variable & Complete responders $(N=13)$ & Partial and nonresponders $(N=27)$ & Test value* \\
& Mean \pm SD & $p$ van \pm SD & -1.442 \\
C3 & $101.15 \pm 22.27 \mathrm{mg} / \mathrm{dL}$ & $91.48 \pm 18.66 \mathrm{mg} / \mathrm{dL}$ & 0.286 \\
C4 & $26.15 \pm 5.51 \mathrm{mg} / \mathrm{dL}$ & $26.67 \pm 5.22 \mathrm{mg} / \mathrm{dL}$ & 0.157 \\
Creatinine & $1.12 \pm 0.24 \mathrm{mg} / \mathrm{dL}$ & $2.53 \pm 0.81 \mathrm{mg} / \mathrm{dL}$ & 0.776 \\
eGFR & $80.15 \pm 24.09 \mathrm{~mL} / \mathrm{min} / 1.73 \mathrm{~m}^{2}$ & $36.59 \pm 10.63 \mathrm{~mL} / \mathrm{min} / 1.73 \mathrm{~m}^{2}$ & 0.0001 \\
Pr/Cr ratio & $1.52 \pm 0.68 \mathrm{mg} / \mathrm{mg}$ & $1.38 \pm 0.58 \mathrm{mg} / \mathrm{mg}$ & -7.994 \\
UNGAL & $13.38 \pm 5.83 \mathrm{ng} / \mathrm{mL}$ & $35.23 \pm 13.47 \mathrm{ng} / \mathrm{mL}$ & -0.701 \\
\hline
\end{tabular}

*Student's $t$-test: significant at 0.05 level. C3, complement component 3; C4, complement component 4; eGFR, estimated glomerular filtration rate; $\mathrm{Pr} / \mathrm{Cr}$ ratio, protein-to-creatinine ratio; UNGAL, urinary neutrophil gelatinase-associated lipocalin.

TABLE 7: Cutoff levels for three-month follow-up UNGAL and conventional biomarkers in predicting renal response to induction.

\begin{tabular}{|c|c|c|c|c|c|c|}
\hline Variable & AUC & Cutoff point & Sensitivity & Specificity & PPV & NPV \\
\hline Creatinine & 0.966 & $1.3 \mathrm{mg} / \mathrm{dL}$ & 92.31 & 96.3 & 92.3 & 96.3 \\
\hline eGFR & 0.979 & $51 \mathrm{~mL} / \mathrm{min} / 1.73 \mathrm{~m}^{2}$ & 92.3 & 92.6 & 85.7 & 96.2 \\
\hline UNGAL & 0.966 & $17.4 \mathrm{ng} / \mathrm{mL}$ & 92.3 & 96.3 & 92.3 & 96.3 \\
\hline
\end{tabular}

eGFR, estimated glomerular filtration rate; UNGAL, urinary neutrophil gelatinase-associated lipocalin.

Diagnostic reliability of the UNGAL in our study showed a good performance of UNGAL in predicting renal response to therapy, discriminating the complete response group from partial and nonresponse groups. As regards baseline UNGAL, ROC analysis showed an AUC value of 0.943 that was greater than those for other parameters (proteinuria, serum creatinine, and estimated GFR). Thus, it outperformed conventional biomarkers; the best cut-off value to discriminate the complete response group from partial and nonresponse groups was $26.5 \mathrm{ng} / \mathrm{mL}$ ( sensitivity $=92.31 \%$ and specificity $=88.89 \%$ ). These results mean that baseline UNGAL can be used with a lot of trust and with a very good predictive performance. Consequently, it may have the potential to predict poor response to induction therapy and perform better than conventional markers in predicting clinical response to treatment of active LN. We investigated the predictive performance of the three-month follow-up UNGAL in comparison to conventional biomarkers. We found better predictive performance of the three-month UNGAL compared to C3. While compared to other conventional biomarkers (serum creatinine and GFR), UNGAL was 


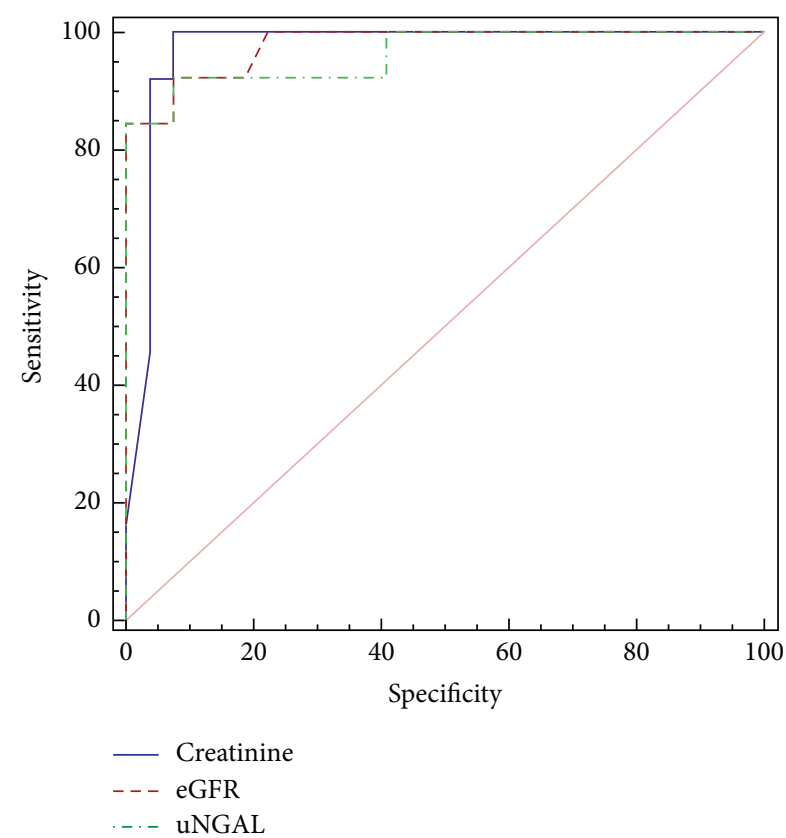

Figure 2: Graph ROC curves showing area under the curve of three-month follow-up UNGAL and conventional biomarkers to predict renal response to induction. It showed an AUC value of 0.966 , with slight differences compared to other conventional biomarkers.

found with an area under the curve value of 0.966 , with slight difference compared to other conventional biomarkers. The best cutoff value for the three-month UNGAL that discriminate the complete response group from partial and nonresponse groups was $17.4 \mathrm{ng} / \mathrm{mL}$ $($ sensitivity $=92.3 \%$ and specificity $=96.3 \%)$.

\section{Conclusion}

This study showed excellent diagnostic performance of UNGAL compared to conventional markers for predicting disease severity and clinical response to treatment of active LN so that it may become one of the most promising biomarkers in $\mathrm{LN}$ patients.

\section{Limitations of the Study}

Our study carries the limitation of a relatively short followup period of 6 months to demonstrate the further rise in serum creatinine or initiating long-term dialysis.

\section{Data Availability}

The data used to support the findings of this study are included within the article.

\section{Ethical Approval}

Ethical issues (including plagiarism, data fabrication, and double publication) have been completely observed by the authors.

\section{Conflicts of Interest}

The authors declare that they have no conflicts of interest.

\section{Authors' Contributions}

All authors contributed to the design of the research. MAA, AMT, MSH, SMS, and WAB were involved in the analysis and interpretation of data. MAA drafted the first version. MAA, AMT, MSH, SMS, and WAB edited the first draft. All authors reviewed, commented on, and approved the final draft.

\section{Acknowledgments}

The authors would like to express their thanks to the staff working in the outpatient clinic and all the patients and their families who helped them to perform this study.

\section{References}

[1] B. Thong and N. J. Olsen, "Systemic lupus erythematosus diagnosis and management," Rheumatology, vol. 56, no. suppl_1, pp. i3-13, 2016.

[2] N. Schwartz, T. Rubinstein, L. C. Burkly et al., "Urinary Tweak as a biomarker of lupus nephritis: a multicenter cohort study," Arthritis Research \& Therapy, vol. 11, no. 5, p. R143, 2009.

[3] G. K. Bertsias, J. E. Salmon, and D. T. Boumpas, "Therapeutic opportunities in systemic lupus erythematosus: state of the art and prospects for the new decade," Annals of the Rheumatic Diseases, vol. 69, no. 9, p. 1603, 2010.

[4] M. Petri, A. M. Orbai, G. S. Alarcón et al., "Derivation and validation of the Systemic Lupus International Collaborating Clinics classification criteria for systemic lupus erythematosus," Arthritis \& Rheumatology, vol. 64, no. 8, pp. 2677-2686, 2012.

[5] G. S. Alarcón, "Multiethnic lupus cohorts: what have they taught us?” Reumatología Clínica, vol. 7, no. 1, pp. 3-6, 2011.

[6] S. Almaani, B. H. Meara, and B. H. Rovin, "Update on lupus nephritis," Clinical Journal of the American Society of $\mathrm{Ne}$ phrology, vol. 12, no. 5, pp. 825-835, 2017.

[7] G. Giannico and A. B. Fogo, "Lupus nephritis: is the kidney biopsy currently necessary in the management of lupus nephritis?" Clinical Journal of the American Society of Nephrology, vol. 8, no. 1, p. 138, 2013.

[8] R. Misra and R. Gupta, "Biomarkers in lupus nephritis," International Journal of Rheumatic Diseases, vol. 18, no. 2, p. 219, 2015.

[9] B. Satirapoj, C. C. Nast, and S. G. Adler, "Novel insights into the relationship between glomerular pathology and progressive kidney disease," Advances in Chronic Kidney Disease, vol. 19, no. 2, p. 93, 2012.

[10] J. B. Cowland and N. Borregaard, "Molecular characterization and pattern of tissue expression of the gene for neutrophil gelatinase-associated lipocalin from humans," Genomics, vol. 45, no. 1, p. 17, 1997.

[11] J. Mishra, Q. Ma, C. Kelly et al., "Kidney NGAL is a novel early marker of acute injury following transplantation," Pediatric Nephrology, vol. 21, no. 6, p. 856, 2006.

[12] A. Sirisopha, S. Vanavanan, A. Chittamma et al., "Effects of therapy on urine neutrophil gelatinase-associated lipocalin in nondiabetic glomerular diseases with proteinuria," 
International Journal of Nephrology, vol. 2016, p. 9, Article ID 4904502, 2016.

[13] H. I. Brunner, M. Mueller, C. Rutherford et al., "Urinary neutrophil gelatinase-associated lipocalin as a biomarker of nephritis in childhood-onset systemic lupus erythematosus," Arthritis \& Rheumatism, vol. 54, no. 8, p. 2577, 2006.

[14] D. D. Gladman, D. Ibañez, and M. B. Urowitz, "Systemic lupus erythematosus disease activity index 2000," The Journal of Rheumatology, vol. 29, no. 2, pp. 288-291, 2002.

[15] H. A. Austin 3rd, L. R. Muenz, K. M. Joyce, T. T. Antonovych, and J. E. Balow, "Diffuse proliferative lupus nephritis: identification of specific pathologic features affecting renal outcome," Kidney International, vol. 25, no. 4, p. 689, 1984.

[16] C.-C. Liu, S. Manzi, and J. M. Ahearn, "Biomarkers for systemic lupus erythematosus: a review and perspective," Current Opinion in Rheumatology, vol. 17, no. 5, p. 543, 2005.

[17] K. Mori, H. T. Lee, D. Rapoport et al., "Endocytic delivery of lipocalin-siderophore-iron complex rescues the kidney from ischemia-reperfusion injury," Journal of Clinical Investigation, vol. 115, no. 3, p. 610, 2005.

[18] T. Rubinstein, M. Pitashny, B. Levine et al., "Urinary neutrophil gelatinase-associated lipocalin as a novel biomarker for disease activity in lupus nephritis," Rheumatology, vol. 49, no. 5, p. $960,2010$.

[19] A. Zickert, B. Sundelin, E. Svenungsson, and I. Gunnarsson, "Role of early repeated renal biopsies in lupus nephritis," Lupus Science \& Medicine, vol. 1, Article ID e000018, 2014.

[20] B. Satirapoj, C. Kitiyakara, A. Leelahavanichkul, Y. Avihingsanon, and O. Supasyndh, "Urine neutrophil gelatinase-associated lipocalin to predict renal response after induction therapy in active lupus nephritis," BMC Nephrology, vol. 18, no. 1, p. 263, 2017.

[21] M. S. El Shahawy, M. H. Hemida, H. A. Abdel-Hafez, T. Z. ElBaz, A.-W. M. Lotfy, and T. M. Emran, "Urinary neutrophil gelatinase-associated lipocalin as a marker for disease activity in lupus nephritis," Scandinavian Journal of Clinical and Laboratory Investigation, vol. 78, no. 4, p. 264, 2018.

[22] S. M. Alharazy, N. C. T. Kong, M. Mohd, S. A. Shah, A. H. Abdul Gafor, and A. Ba'in, "The role of urinary neutrophil gelatinase-associated lipocalin in lupus nephritis," Clinica Chimica Acta, vol. 425, pp. 163-168, 2013.

[23] H. Batool, T. Naseem, and M. Shoukat, "Correlation of urinary neutrophil gelatinase associated lipocalin with routine biomarkers used to detect kidney injury in systemic lupus erythematosus patients with and without nephritis," Annals of King Edward Medical University, vol. 23, no. 3, 2017. 